\title{
The overexpression of the Arabidopsis $N D B 2$ gene in tobacco plants affects the expression of genes encoding the alternative mitochondrial electron transport pathways and stress proteins
}

\author{
Borovskii G.B. ${ }^{1 *}$, Korotaeva N.E. ${ }^{1}$, Katyshev A.I. ${ }^{1}$, Fedoseeva I.V. ${ }^{1}$, Fedyaeva A.V. ${ }^{1}$, \\ Kondakova M.A. ${ }^{1}$, Rikhvanov E.G. ${ }^{1}$, Shyshlova-Sokolovskaya A.M. ${ }^{2}$, Sauchyn D.G. ${ }^{2}$, \\ Urbanovich O.Yu. ${ }^{2}$ \\ ${ }^{1}$ Siberian Institute of Plant Physiology and Biochemistry, SB RAS, Irkutsk, Russia \\ ${ }^{2}$ Institute of Genetics and Cytology of NAS of Belarus, Minsk, Belarus \\ *email: borovskii@sifibr.irk.ru
}

An important feature of the plant stress response is the activation of alternative pathways of mitochondrial electron transport chain, in particular, rotenone-insensitive NAD(P)Hdehydrogenases. The exact physiological function of its protein family has not been established. It is believed that, together with an alternative oxidase, they participate in the formation of a non-phosphorylating respiratory chain under oxidative stress and suppress the generation of reactive oxygen species. It was proposed that, rotenoneinsensitive $\mathrm{NAD}(\mathrm{P}) \mathrm{H}$-dehydrogenases, together with the alternative oxidase, participate in formation of a non-phosphorylating electron transport chain in response to oxidative stress and suppress the reactive oxygen species generation. An alternative hypothesis suggests that its proteins are important sources of ROS generation. It is assumed that the enzyme of "external" non-phosphorylating NADH dehydrogenase (NDB2) affects the mitochondrial functioning, expression of stress response genes, and stress signaling program of by changing the ROS amount. The genetically modified Nicotiana tabacum plants expressing NDB2 from Arabidopsis thaliana were obtained to elucidate the role of its protein in the plant resistance mechanisms. In the leaves of T4 plants, $n d b 2$ expression and accumulation of the A. thaliana NDB2 protein were shown. AtNDB2 expression and accumulation and of corresponding protein and its localization in tobacco mitochondria were shown. AtNDB2 overexpression changes the expression of tobacco genes involved in alternative electron transport pathways, affects the stress proteins content, comparative rate of ROS generation and the level of TBA-reactive products in tobacco leaves. It can be assumed that AtNDB2 influences the stress response, the development of oxidative stress, and tobacco tolerance to low temperatures. 